\title{
DEVELOPMENT AND EVALUATION OF LAMOTRIGINE SOYA LECITHIN SOLID DISPERSION: IN VITRO AND PHARMACODYNAMIC INVESTIGATION
}

\author{
SURESH GAUTAM, YOGESH NIKALAJE, DARSHANA BHADRE, RASHMI TRIVEDI, MILIND UMEKAR, JAYSHREE \\ TAKSANDE*
}

Department of Pharmaceutics, Shrimati Kishoritai Bhoyar College of Pharmacy, New Kamptee, Nagpur (M. S.), India 441002

Email: jayabtaksande@gmail.com

Received: 10 Sep 2019, Revised and Accepted: 15 Nov 2019

\begin{abstract}
Objective: Epilepsy is a common neurodegenerative disorder characterized by spontaneous and repeated attacks of convulsions. It requires immediate pharmacotherapy to prevent its progression to status epilepticus. However, most of the anticonvulsant drugs are poorly water-soluble and demonstrate the delayed onset of action. Thus there is a need to improve its solubility for the better pharmaceutical profile. The objective of the present investigation was to enhance the solubility of lamotrigine incorporating soya lecithin as a phospholipid carrier by solid dispersion technique
\end{abstract}

Methods: Solid dispersions of lamotrigine were prepared with soya lecithin by the solvent method. The effect of concentration of phospholipid and solvents on aqueous solubility and dissolution profile of lamotrigine was analyzed.

Results: Ethanol increased lamotrigine solubility with soya lecithin in ratio 5:1. X-ray diffraction and scanning electron micrograph indicated a smaller crystallite size of lamotrigine with fairly uniform size distribution in the lamotrigine-soya lecithin solid dispersion. The resultant solid dispersion also significantly delayed the onset of clonic convulsion (875.8 s) as compared to control (85.5 s) and offered complete protection $(100 \%)$ against the pentylenetetrazole induced seizures in the rat as compared to control (33.33\%). Also, solid dispersion with maximum drug content $(77.68 \%)$ and dissolution rate $(91.40 \%)$ was formulated as an orodispersible tablet and characterized for its pharmaceutical properties.

Conclusion: It can be concluded that the solid dispersion of lamotrigine incorporated with soya lecithin demonstrated enhanced solubility and dissolution rate may have potential clinical application.

Keywords: Lamotrigine, Phospholipids, Solid dispersion, Anticonvulsant activity, Pentylenetetrazole

(C) 2020 The Authors. Published by Innovare Academic Sciences Pvt Ltd. This is an open-access article under the CC BY license (http://creativecommons.org/licenses/by/4.0/) DOI: http://dx.doi.org/10.22159/ijap.2020v12i1.35647. Journal homepage: https://innovareacademics.in/journals/index.php/ijap

\section{INTRODUCTION}

Epilepsy is a very common neurodegenerative disorder characterized by repeated episodes of epileptic attacks. It required immediate medical help to prevent its progression to status epilepticus [1]. Although pharmacological treatment has been more pronounced due to the availability of new generation anticonvulsant drugs, some of them are poorly water-soluble and show delayed onset of action following their oral administration [2]. Low aqueous solubility is the major problem encountered with the formulation development of such drugs [3]. If the rate of dissolution of the drug is significantly slower than the rate of absorption, the dissolution of the drug becomes the rate-limiting step in the absorption process. Different formulation approaches have been used to improve the oral absorption of drugs with low water solubility by increasing the dissolution rate and solubility $[4,5]$.

Lamotrigine is a second-generation antiepileptic drug that shows a broad spectrum of actions against partial, generalized tonic-clonic seizures and Lennox-Gastaut syndrome either alone or as adjunctive therapy [6, 7]. Comparatively, lamotrigine shows relatively few side effects and does not require blood monitoring in monotherapy $[8,9]$. However, peak plasma concentration reaches after $1.4 \mathrm{~h}$ its oral administration. This delay in the onset of action in spite of good bioavailability is due to its low aqueous solubility $(0.17 \mathrm{~g} / \mathrm{l})[2,8]$. Hence it is required to improve the solubility and dissolution of lamotrigine.

Enhancement of the solubility of poorly water drugs is a difficult task. Several methods have been used for solubility enhancement such as salt formation [10], complexation [11], solid dispersion [12], microcrystals [13], microemulsion [14] etc. One successful approach to enhance the solubility is solid dispersions using an appropriate carrier. The dissolution rates may be improved by dispersing drugs in polymeric carriers by fusion and solvent evaporation methods [3]. Though various solid dispersion techniques are available, the solvent method is advantageous as it requires low temperature for the evaporation of organic solvents that prevents the decomposition of drugs and carriers [15].
The preparation of solid dispersion using soluble carriers increases the solubility and dissolution rate of the drug, as it is exposed to the dissolution medium in the very fine particulate form $[8,16]$. Hydrophilic polymers have been used to enhance the solubility and dissolution rate of lamotrigine. Some of the investigators have prepared and evaluated the formulations of solid dispersion of lamotrigine using hydrophilic polymers $[8,17]$. However, it requires a great amount of the carrier for the preparation of solid dispersion. Thus it would be useful to use a minimum quantity of carriers such as phospholipids as solubility enhancers for the preparation of solid dispersion. Phospholipids are required in much lower concentration for increasing the dissolution rate of poorly water-soluble drugs. These forms spherical bilayer structures (liposomes) when it is dispersed in aqueous media into which the drug is entrapped or sequestered [18-20] and released rapidly into the dissolution medium. Besides the assimilation of drugs with lipids or lipid-like compounds promotes the oral absorption of the drug by intrinsic lipid pathway [21].

Soya lecithin, the phospholipid is a multi-functional surface-active agent having various applications. Phospholipids due to their amphiphilic property have the solubilizing potential for poorly water-soluble drugs $[22,23]$ can be used as a carrier for the preparation of solid dispersion. With this background, the present investigation was carried out to enhance the solubility and dissolution of lamotrigine incorporating soya lecithin as a phospholipid carrier by solid dispersion technique. Also to determine the anticonvulsant activity of prepared formulation in rats and to develop an orally disintegrating tablet containing solid dispersion to achieve fast release of drug from the dosage form.

\section{MATERIALS AND METHODS}

\section{Materials}

Lamotrigine was obtained as a gift sample from Glenmark, Mumbai, soya lecithin was purchased from Himedia Lab. Mumbai, DMSO, and methanol were procured from Merck chemicals, Mumbai. Ethanol 
was procured from Changshu Hongsheng Fine Chemical Co. Ltd Jiangsu Province. Chloroform was purchased from Loba Chemie Mumbai, croscarmellose sodium from Akhil Healthcare Mumbai and cross povidone was obtained from Amnem Mumbai, sodium starch glycolate was purchased from Sigma Aldrich, Mumbai. All the chemicals used in experiments were of analytical grade.

Preparation of phospholipid solid dispersions and physical mixture

Solid dispersions of lamotrigine and phospholipids were prepared by the solvent method $[24,25]$ using chloroform, DMSO, and ethanol as solvent. Weighed amounts of phospholipid and lamotrigine (ratio of 1:1, 1:3, 1:5 and 1:10) were added to the solvent and dissolved with gentle stirring further sonicated for $30 \mathrm{~min}$ after complete dissolution solvent was removed at room temperature or up to $60^{\circ} \mathrm{C}$ temperature if required. Further drying was accomplished in a vacuum desiccator overnight. Physical mixtures were prepared by gently triturating appropriate quantities of lamotrigine and phospholipid using a mortar and pestle. Solid dispersions and physical mixtures were passed through an 80-mesh sieve to obtained uniform size powder for further studies. The formulation composition for solid dispersion is depicted in table 1.

Table 1: Composition of lamotrigine solid dispersion

\begin{tabular}{|c|c|c|c|c|c|c|c|c|c|c|c|c|}
\hline Ingredients & S1 & $\mathbf{S 2}$ & S3 & S4 & S5 & S6 & S7 & S8 & S9 & S10 & S11 & S12 \\
\hline PLP: LTG & $1: 1$ & $1: 3$ & $1: 5$ & $1: 10$ & $1: 1$ & $1: 3$ & $1: 5$ & $1: 10$ & $1: 1$ & $1: 3$ & $1: 5$ & $1: 10$ \\
\hline DMSO+Ethanol (ml) (2:1) & 10 & 10 & 10 & 10 & - & - & - & - & - & - & - & - \\
\hline Ethanol ml) & - & - & - & - & 10 & 10 & 10 & 10 & - & - & - & - \\
\hline Chloroform+ Ethanol (ml) $(2: 1)$ & - & - & - & - & - & - & - & - & 10 & 10 & 10 & 10 \\
\hline
\end{tabular}

\section{Saturation solubility}

Saturation solubility was performed, adding an excess of the amount of sample in $20 \mathrm{ml}$ distilled water in a screw-capped flask and shaken in a rotary flask shaker $(20 \mathrm{rpm})$ at room temperature $\left(37 \pm 0.5^{\circ} \mathrm{C}\right)$ for $48 \mathrm{~h}$. Once equilibrium was achieved appropriate aliquots were withdrawn and filtered through Whatman filter paper no. 45 . The filtrate was analyzed for drug spectrophotometrically at $307 \mathrm{~nm}[26]$.

\section{Drug content}

Drug content was determined by dissolving solid dispersion or physical mixture equivalent to $10 \mathrm{mg}$ of drug in $10 \mathrm{ml}$ methanol following ultra-sonication for $20 \mathrm{~min}$. The volume was adjusted to $100 \mathrm{ml}$ with a $6.8 \mathrm{pH}$ buffer. The solution was filtered through Whatman filter paper no. 45 , further suitably diluted with $6.8 \mathrm{pH}$ buffer and the absorbance was measured at $307 \mathrm{~nm}$ using a doublebeam UV spectrophotometer [27].

\section{Dissolution studies}

The dissolution studies were carried out using the TAB dissolution test apparatus (Cambell DRS Company). The dissolution flasks were immersed in the water bath equipped with an external temperature control unit. $10 \mathrm{mg}$ of the sample was dispersed on the surface of the dissolution medium at the beginning of the study. A USP standard paddle continuously stirred the dissolution medium $(900 \mathrm{ml}$ of 6.8 $\mathrm{pH}$ buffer) at $100 \mathrm{rpm}$ at $37 \pm 0.5^{\circ} \mathrm{C}$. A sink condition was maintained in the dissolution medium. Samples were taken at pre-determined intervals and the concentration of lamotrigine present in the dissolution medium was determined using a double-beam UV spectrophotometer at $307 \mathrm{~nm}$ [25].

\section{Characterization of lamotrigine phospholipid solid dispersions}

\section{Differential scanning calorimeter (DSC)}

The DSC analysis was performed using Mettler Toledo DSC822e using aluminum crucibles with about $6.5-10 \mathrm{mg}$ of samples, under dynamic $\mathrm{N}_{2}$ atmosphere $\left(10 \mathrm{ml} \mathrm{min}{ }^{-1}\right)$ and heating rate of $20{ }^{\circ} \mathrm{C}$ $\mathrm{min}^{-1}$ under a temperature range from $25^{\circ} \mathrm{C}$ to $350^{\circ} \mathrm{C}$.

\section{Powder X-ray diffraction studies (PXRD)}

X-ray diffraction study was performed to determine the crystallinity of the prepared solid dispersion using Bruker X-ray diffractometer AXS D8 Advance using a Cu radiation (1.5406 $\AA$ ) at a voltage $2.2 \mathrm{kV}$, and Detector-Si(Li)PSD. Diffractogram was scanned in the range from $3{ }^{\circ} \mathrm{C}$ to $80^{\circ} \mathrm{C}(2 \theta)$ with a resolution of $0.02{ }^{\circ} \mathrm{C}$ and scanning speed of $2.0^{\circ} \mathrm{C} \mathrm{min}-1$.

\section{Fourier-transform infrared spectroscopy}

Fourier-transform infrared spectroscopy (FTIR) spectrum was recorded on Thermo Nicolet, Avatar 370 using the potassium bromide pellet method. About $2 \mathrm{mg}$ of the sample was mixed with potassium bromide, and the mixture was compressed at a pressure of 5 tons for $5 \mathrm{~min}$ in a hydraulic press to form the pellets. The sample was scanned at $4 \mathrm{~cm}^{-1}$ resolution.

\section{Scanning electron microscopy (SEM)}

A JSM-6390LV field emission scanning electron microscope (JEOL, Peabody, MA) was used to determine the particle shape and size of lamotrigine and solid dispersion. Photomicrograph was taken at $1500 x$ to $3500 x$ magnification.

\section{Pharmacodynamic study}

The study was carried out to investigate the anticonvulsant effect of an optimized batch of solid dispersion in PTZ induced seizures in rats through oral administration. It is commonly used in preclinical paradigms for predicting the anti-convulsant activity of lamotrigine after their acute administration. Adult male Sprague Dawley rats (National Institute of Nutrition; Hyderabad, India) weighing 200-280 g were housed in standard laboratory conditions of temperature $\left(23 \pm 1^{\circ} \mathrm{C}\right)$ and relative humidity $(55 \pm 5 \%)$, with free access to food (NIN, Hyderabad, India) and water. Animals were divided into different groups $(n=4)$, fasted overnight before the experiments and transferred to the laboratory at least $1 \mathrm{~h}$ before the beginning of the experiment. The experiments were performed during the light cycle in between 9.00 to $13.00 \mathrm{~h}$. All the experimental protocols were approved by the Institutional Animal Ethics Committee (853/IAEC/201819/25). Oral doses of solid dispersion (equivalent to lamotrigine: $5 \mathrm{mg} / \mathrm{kg}, 10 \mathrm{mg} / \mathrm{kg}$ ) and pure drug in saline were administered using a polyethylene tube. The tube was inserted about 5-6 $\mathrm{mm}$ deep for the proper delivery of drugs orally. $1 \mathrm{~h}$ following drug administration, animals were subcutaneously injected with PTZ $(60 \mathrm{mg} / \mathrm{kg})$ and the onset of clonic-tonic convulsions and percentage protection in each group were recorded.

\section{Development of orodispersible tablet}

For the preparation of oral dispersible tablet using solid dispersion formulation with maximum drug content and better dissolution profile was selected. The formulation composition of lamotrigine orodispersible tablets is depicted in table 2 . Tablets were prepared by the direct compression method by taking solid dispersion equivalent to $25 \mathrm{mg}$ of lamotrigine. Microcrystalline cellulose (MCC) and mannitol were used as directly compressible agents. Sodium starch glycolate (SSG), crospovidone (CPD), croscarmellose sodium (CCS) were used as super disintegrant agents in different batches. Aspartame was added as the sweetening agent. Talc and magnesium stearate were added as lubricant and glidant and compressed as a tablet using flat-faced punches. The hardness of the tablets was kept constant and was measured with a hardness tester. Tablet blend and tablets were evaluated for various pre-compression and postcompression parameters [17]. 
Table 2: Composition of lamotrigine orodispersible tablets

\begin{tabular}{|c|c|c|c|c|c|c|}
\hline Ingredients (mg) & F1 & F2 & F3 & F4 & F5 & F6 \\
\hline SD LTG & 26.05 & 26.05 & 26.05 & 26.05 & 26.05 & 26.05 \\
\hline SSG & 6 & 8 & - & - & - & - \\
\hline CCS & - & - & 6 & 8 & - & - \\
\hline CPD & - & - & - & - & 6 & 8 \\
\hline Mannitol & 49.15 & 47.45 & 49.15 & 47.45 & 49.15 & 47.45 \\
\hline MCC & 8.5 & 8.5 & 8.5 & 8.5 & 8.5 & 8.5 \\
\hline Aspartame & 3 & 3 & 3 & 3 & 3 & 3 \\
\hline Talc & 3 & 3 & 3 & 3 & 3 & 3 \\
\hline Aerosil & 2 & 2 & 2 & 2 & 2 & 2 \\
\hline Mg stearate & 2 & 2 & 2 & 2 & 2 & 2 \\
\hline Total Weight & 100 & 100 & 100 & 100 & 100 & 100 \\
\hline
\end{tabular}

\section{Statistical analysis}

All the results are reported as mean \pm standard deviation (SD) or mean \pm standard error mean (SEM). Statistical comparisons were performed by one-way analysis of variance followed by Bonferroni's multiple comparison test. Differences between formulations were considered to be statistically significant at $P<0.05$ in all cases.

\section{RESULTS AND DISCUSSION}

\section{Preparation and characterization of lamotrigine solid dispersion}

Solid dispersion of lamotrigine-soya lecithin was prepared by the solvent method. Preformulation studies were performed for lamotrigine solubility in ethanol, chloroform, DMSO, ethanolchloroform and DMSO-ethanol to select the solvent system for the preparation of solid dispersion. Based on the results solid dispersions were prepared by using DMSO-ethanol (2:1), chloroform-ethanol (2:1) and ethanol as solvents. The aqueous solubility of pure lamotrigine was found to be $0.1515 \mathrm{mg} / \mathrm{ml}$. Solid dispersions were prepared by using the various ratio of lamotrigine and soya lecithin using different solvent were analyzed for aqueous solubility. Results revealed an increase in solubility was for the batch S7 prepared by using ethanol as a solvent in comparison to other solvents. Solid dispersion prepared with DMSO and ethanol showed an increase in solubility of lamotrigine as compared to that of the pure drug, but the preparation was more viscous and difficult to dry. Batches prepared with ethanol and chloroform as solvent showed an increase in aqueous solubility, but results were more enhanced for the solid dispersions prepared with ethanol as solvent. Batch S7 consisting of soya lecithin and lamotrigine in ratio 1:5 showed a significant increase in aqueous solubility of lamotrigine i.e. $3.1814 \mathrm{mg} / \mathrm{ml}$ as compared to its lower ratios(1:1 and 1:3). However, a further increase in ratio showed a decreased solubility. A physical mixture of lamotrigine with soya lecithin showed an increase in aqueous solubility than pure drug, but it was very less as compared to aqueous solubility of various solid dispersions. Table 3 depicts the results for the effect of solvents and the ratio of phospholipid and lamotrigine on the saturation solubility of lamotrigine.

The drug content of the formulation was ranging from $41.39 \%$ to $77.68 \%$, indicating maximum entrapment of drug in the formulation. The maximum amount of drug content $77.68 \pm 1.35 \%$ was obtained for solid dispersion with a 1:5 ratio of soya lecithin and lamotrigine using ethanol as solvent. The percentage of drug content proportionally increased with the drug concentration.

Table 3: Saturation solubility of lamotrigine, solid dispersions and physical mixture

\begin{tabular}{llll}
\hline Formulation code & Media & Solubility $(\mathbf{m g} / \mathbf{m l})$ & Drug content*(\% w/w) \\
\hline LTG & 6.8 Buffer & 0.4882 & - \\
LTG & Ethanol & 1.8232 & - \\
LTG & water & 0.1515 & - \\
S1 & water & 1.6501 & $41.39 \pm 0.77$ \\
S2 & water & 1.7528 & $49.84 \pm 1.68$ \\
S3 & water & 1.8804 & $61.69 \pm 1.75$ \\
S4 & water & 2.2552 & $69.37 \pm 0.57$ \\
S5 & water & 0.1942 & $39.83 \pm 1.95$ \\
S6 & water & 2.9986 & $57.46 \pm 5.90$ \\
S7 & water & 3.1814 & $77.68 \pm 1.35$ \\
S8 & water & 3.0400 & $72.55 \pm 1.27$ \\
S9 & water & 1.9821 & $40.4 \pm 1.94$ \\
S10 & water & 2.0447 & $50.84 \pm 0.86$ \\
S11 & water & 2.2356 & $70.32 \pm 0.64$ \\
S12 & water & 2.3252 & $68.56 \pm 1.16$ \\
PM & water & 0.1787 & $62.7 \pm 1.06$ \\
\hline
\end{tabular}

Results are shown as mean $\pm \operatorname{SD}(* n=3)$

\section{Dissolution studies}

The lamotrigine-soya lecithin solid dispersions prepared from ethanol exhibited greater dissolution as particles formed were fine, non-waxy and discrete. Fig. 1 shows the effect of different ratios of lamotrigine to soya lecithin on the dissolution of lamotrigine. Increasing the soya lecithin content in this system from 5:1 to $1: 1$ showed a significant increase of $96.68 \pm 0.41 \%$ in the extent of dissolution at $75 \mathrm{~min}$; also the initial rate of dissolution at 5 min was increased. However, the results were slightly decreased for the system containing lamotrigine and soya lecithin ratio 10:1. There were no significant differences in dissolution between 5:1 and 10:1 ratios of lamotrigine to soya lecithin. This illustrates that a small amount of the carrier phospholipid was adequate for a significant increase in the rate and extent of dissolution. An increase in dissolution rate was observed for the solid dispersions as compared to physical mixture and lamotrigine. This might be because of the decrease in particle size of the crystallites, resulting in greater wetting and increase in surface area of particles or because the crystallites are coated with the phospholipid or both. When placed into an aqueous medium, phospholipids rapidly form liposomal structures on dispersion resulting in an effective increase in the 
saturation concentration of drugs in the diffusion layer during the dissolution process through the release of substantially entrapped finely dispersed drug from the lipid bilayer or the aqueous compartments. This may be another reason for the increased dissolution of lamotrigine from the lamotrigine-soya lecithin solid dispersion.

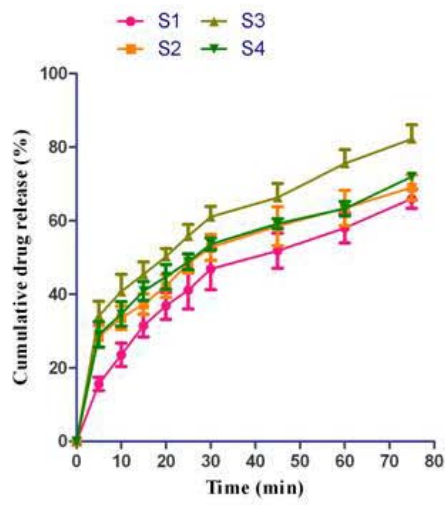

a

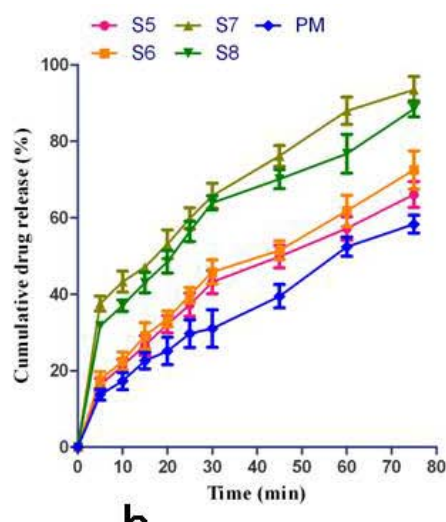

b

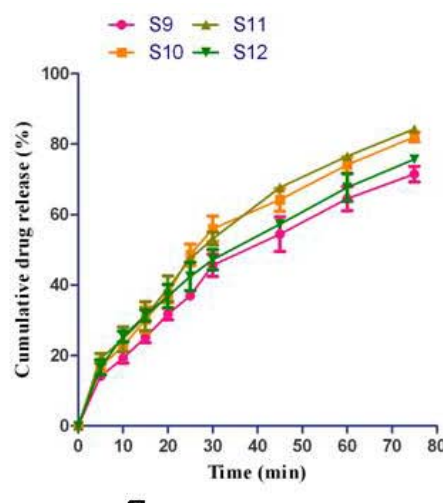

C

Fig. 1: Dissolution profile of a) DMSO and ethanol (2:1) treated solid dispersions of lamotrigine with a phospholipid, b) ethanol-treated solid dispersions of lamotrigine with phospholipid and c) chloroform and ethanol (2:1) treated solid dispersions of lamotrigine with phospholipid in $6.8 \mathrm{pH}$ buffer

\section{Characterization of lamotrigine phospholipid solid dispersions}

\section{Differential scanning calorimeter (DSC)}

DSC thermogram of lamotrigine, lamotrigine-soya lecithin physical mixture and solid dispersion of lamotrigine-soya lecithin prepared by using ethanol are depicted in fig. 2 . The studies revealed the formation of lamotrigine solid dispersion with and broaden endothermic peak at $206.69{ }^{\circ} \mathrm{C}$ by the solvent method. DSC thermograph of pure lamotrigine exhibited a sharp endothermic peak at $217.48{ }^{\circ} \mathrm{C}$ suggests the melting temperature of lamotrigine which has shifted backward and becoming much broader, suggests the formation of crystals of solid dispersion with soya lecithin.

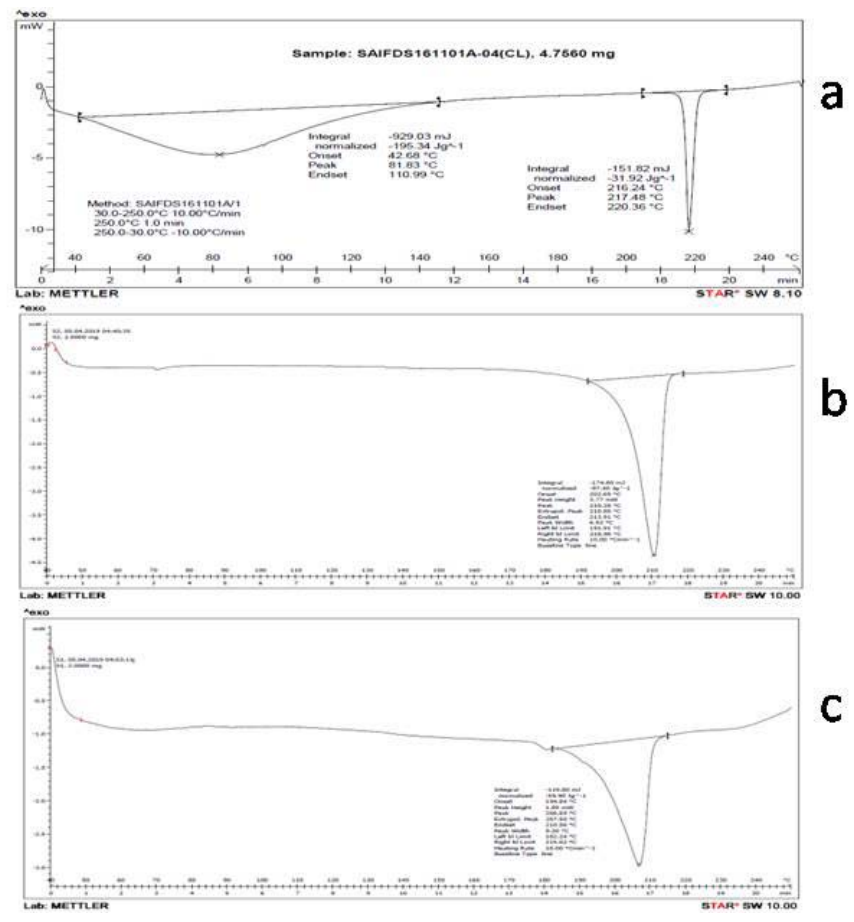

Fig. 2: DSC spectral analysis of a) lamotrigine, b) lamotrigine-soya lecithin physical mixture and c) Solid dispersion of lamotrigine-soya lecithin using ethanol

\section{X-ray diffraction studies (XRD)}

XRD analysis of lamotrigine, lamotrigine-soya lecithin physical mixture and solid dispersion of lamotrigine-soya lecithin prepared by using ethanol are depicted in fig. 3. The X-ray diffractogram of lamotrigine shows a sharp and intense peak indicating the crystalline nature of the drug in pure form. The X-ray diffractogram of the physical mixture and solid dispersion were obtained to determine if there is a loss or modification of the pure drug's crystal structure after it is formed into a solid phospholipid dispersion, and to determine if any new crystalline phases may have formed. Fig. 3 indicates that there is virtually no difference in crystallinity between 
prepared solid dispersion and pure lamotrigine proposed that enhanced dissolution might be due to the increased surface area of the drug crystallites after the formation of the solid dispersions and not due to a change from a crystalline to an amorphous state [25].

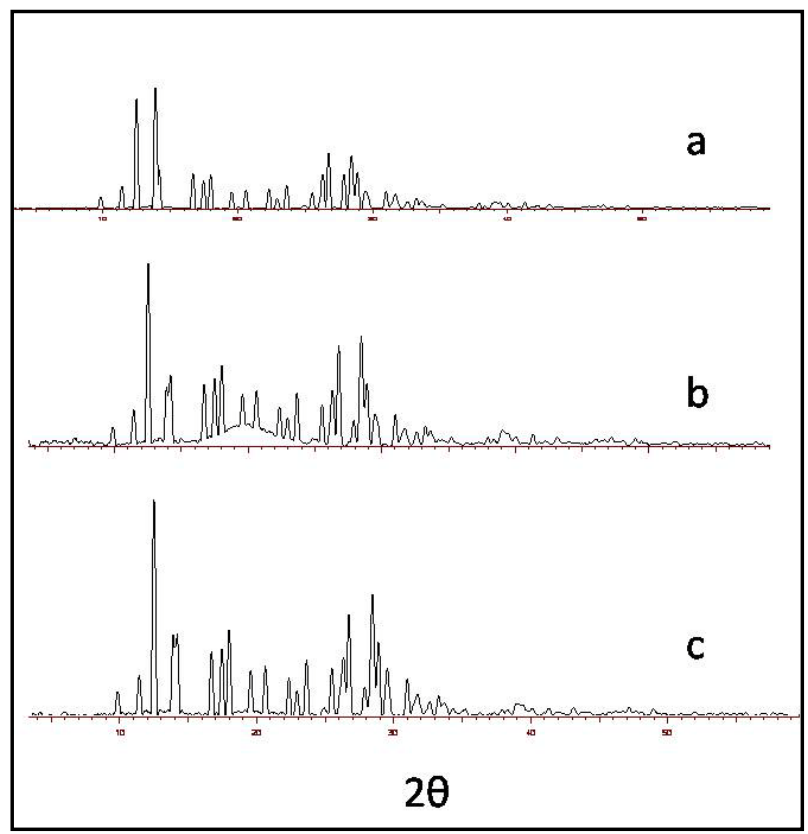

Fig. 3: XRD spectral analysis of a) lamotrigine, b) lamotrigine soya lecithin physical mixture and c) Solid dispersion of lamotrigine using ethanol

\section{FTIR analysis}

Results of FTIR spectral analysis of lamotrigine, soya lecithin, physical mixture of lamotrigine-soya lecithin and solid dispersion of lamotriginesoya lecithin are depicted in fig. 4. FTIR spectra of lamotrigine showed characteristic absorbance at $3448 \mathrm{~cm}^{-1}$ specifying $\mathrm{NH}$ stretching of an amino group (Aromatic), $3209 \mathrm{~cm}^{-1}$ shows aromaticity (aromatic $\mathrm{CH}$ stretching), $1620 \mathrm{~cm}^{-1}, 1486 \mathrm{~cm}^{-1}$ indicates $\mathrm{C}=\mathrm{C}$ ring stretching and 962 $\mathrm{cm}^{-1}$ shows $\mathrm{C}-\mathrm{Cl}$ stretching of halides. In FTIR spectra of physical mixture characteristics peak of both lamotrigine and soya lecithin were observed. It suggests no significant interaction between drug and excipient However, the characteristic absorbance peaks of lamotrigine were found to be shifted to lower values and decreased in intensity also, suggesting the formation of solid dispersion of lamotrigine with soya lecithin by using ethanol as solvent

Thus, based on DSC, XRD, and FTIR analysis, the formation of crystallites of solid dispersion was affirmed.
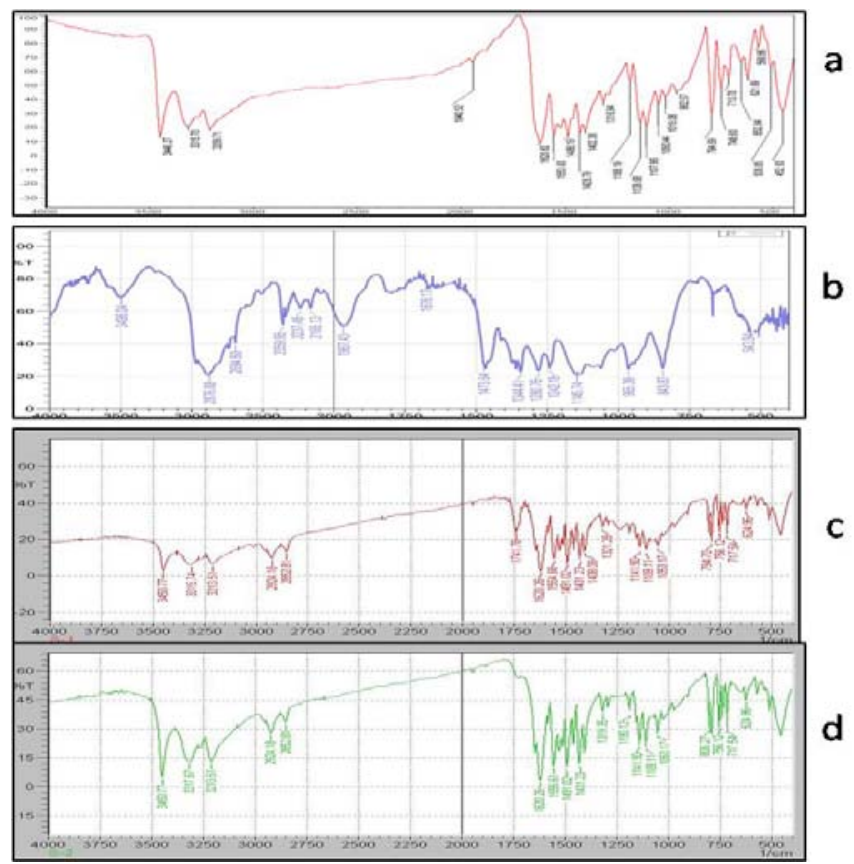

Fig. 4: FTIR spectral analysis of a) lamotrigine, b) soya lecithin, c) lamotrigine soya lecithin physical mixture and d) Solid dispersion of lamotrigine using ethanol 


\section{Scanning electron microscopy (SEM)}

Fig. 5 shows SEM images of the lamotrigine and solid dispersion of lamotrigine-soya lecithin. As observed from the fig. 5 particles of lamotrigine-soya lecithin solid dispersion showed the regular shape and smooth surface and significant reduction of the lamotrigine crystallite size as compared to pure lamotrigine. This allows faster solvating of the drug and the faster breakup of the solid dispersions. This could be because the crystallites are less than a micron in size. This may be the reason for the increased dissolution of lamotrigine from the lamotrigine-soya lecithin solid dispersion.



Fig. 5: SEM image of a) Lamotrigine and b) Solid dispersion of lamotrigine using ethanol

\section{Pharmacodynamic study}

As shown in fig. 6, administration of PTZ to vehicle-treated rat produced clonic convulsions in all animals, and the onset of such convulsions was $85.5 \pm 14.36 \mathrm{~s}$. Oral administration of solid dispersion of lamotrigine $(10 \mathrm{mg} / \mathrm{kg}$ ) significantly delayed the onset of clonic-tonic seizures in PTZ injected animals up to $875.75 \mathrm{~s}$ as compared to administration of lamotrigine powder (634 s). It also offered $100 \%$ protection against the mortality induced by PTZ which was $66.66 \%$ with lamotrigine powder. Similarly, administration of solid dispersion of lamotrigine in dose $5 \mathrm{mg} / \mathrm{kg}$ too delayed the onset of clonic-tonic seizures in PTZ injected animals up to $691.25 \mathrm{~s}$ and provided $100 \%$ protection against mortality induced by PTZ The results indicate the greater anticonvulsant effect of lamotriginesoya lecithin solid dispersion in PTZ-induced seizures as compared to pure lamotrigine. The results of the study are also supported by our earlier observations demonstrating the anticonvulsant effect of lamotrigine in PTZ induced clonic tonic seizures [28, 29].

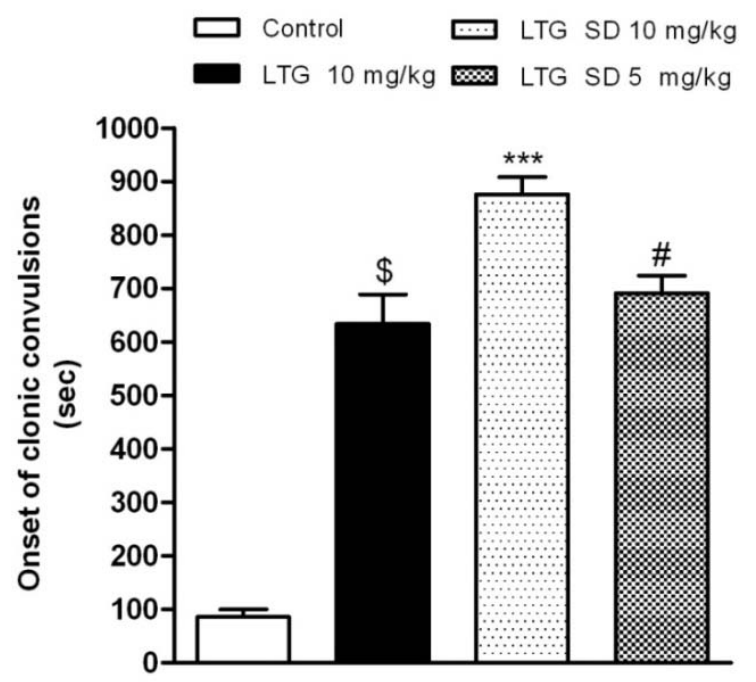

Fig. 6: Effect of lamotrigine solid dispersion on the mean onset time of convulsions against pentylenetetrazole-induced seizures. ${ }^{* * *} \mathrm{P}<0.001$ as compared to control group; $\# \mathrm{P}<0.001$ as compared to the control group. Data are represented as the mean onset time of convulsions \pm SEM. Statistical analysis by ANOVA followed by Bonferroni's multiple comparison test $(n=4)$

\section{Characterization of lamotrigine orodispersible tablets}

\section{Precompression studies}

Solid dispersion of batch S7 consisting of 1:5 ratio of soya lecithinlamotrigine was obtained as discrete, uniform and non-greasy particles showed maximum drug content, better dissolution profile as compared to other batches and significant anticonvulsant activity so it was selected for the formulation of orodispersible tablets. The tablet blend was prepared by using various super disintegrants such as SSG, CCS, and CPD. The powder blends were evaluated for various 
pre-compression parameters of powder blend and results are depicted in table 4 . The bulk density found to be in range $0.523 \pm 0.003$ to $0.558 \pm 0.002 \mathrm{~g} / \mathrm{cm}^{2}$, which was within acceptable limits. The angle of repose of all the formulations was found to be less than $25^{\circ}$ indicated excellent flowability of the powder blend. Another important parameter to determine powder compressibility and flowability is the Hausner's ratio. A ratio greater than 1.5 indicates cohesive and poor flow property of powder blend. Results revealed the values less than 1.5 indicates good flow and compression property of the powder blends. Results of Carr's index also indicate acceptable flow property and compression behavior powder blend to be compacted as tablets.

Table 4: The Pre-compression evaluation parameters of the powder blend

\begin{tabular}{|c|c|c|c|c|c|}
\hline Formulations & Bulk density (g/ml) & Tapped density (g/ml) & Carr's index (\%) & Hausner's ratio & Angle of repose $(\theta)$ \\
\hline F1 & $0.545 \pm 0.001$ & $0.761 \pm 0.003$ & $28.38 \pm 1.23$ & $1.396 \pm 0.025$ & $19.28 \pm 0.65$ \\
\hline $\mathrm{F} 2$ & $0.558 \pm 0.003$ & $0.727 \pm 0.004$ & $26.77 \pm 0.98$ & $1.302 \pm 0.031$ & $19.89 \pm 0.88$ \\
\hline F3 & $0.551 \pm 0.004$ & $0.738 \pm 0.002$ & $25.33 \pm 0.56$ & $1.339 \pm 0.011$ & $20.12 \pm 0.76$ \\
\hline F4 & $0.523 \pm 0.002$ & $0.691 \pm 0.003$ & $21.10 \pm 0.57$ & $1.321 \pm 0.014$ & $20.33 \pm 0.65$ \\
\hline F5 & $0.564 \pm 0.01$ & $0.716 \pm 0.006$ & $23.22 \pm 0.87$ & $1.269 \pm 0.023$ & $19.66 \pm 0.63$ \\
\hline F6 & $0.534 \pm 0.005$ & $0.696 \pm 0.004$ & $22.27 \pm 1.05$ & $1.303 \pm 0.046$ & $19.35 \pm 1.03$ \\
\hline
\end{tabular}

Results are shown as mean $\pm \mathrm{SD}(\mathrm{n}=3)$

\section{Post compression studies}

Orodispersible tablets were evaluated for post-compression properties and results are depicted in table 5. For all formulations, results of weight variation were found to be within limit $100 \pm 1.448$ The hardness test indicated a good mechanical strength of $3.33 \pm 0.87$ $\mathrm{kg} / \mathrm{cm}^{2}$. The friability was obtained to be less than $1 \%$ which indicates that all the batches have good mechanical resistance. The tablets were evaluated for the in vitro disintegration time, results revealed less disintegration time $22.59 \mathrm{~s}$ for the tablets consisting of CCS. The disintegration time was more with SSG which might be due to the formation of a viscous gel layer which leads to a thick barrier to further penetration of the disintegration medium and hindered the disintegration of tablet content. Thus the tablet disintegration is retarded to some extent with the tablet containing SSG when compared with the disintegration time of the tablet containing CCS. The release profile for a formulation containing various super disintegrant and magnesium stearate as a lubricant is depicted in fig. 7. The drug content of the formulation was ranging from $82.31 \%$ to $95.27 \%$ indicating maximum entrapment of drug in the formulation. F1 formulation gives $89.08 \%$ at $15 \mathrm{~min}$ and F3, F4 also shows significant release $96.34 \%, 100.06 \%$ respectively. These results revealed that maximum drug release was observed when lamotrigine was combined with soya lecithin in a 1:5 ratio for solid dispersion prepared by the solvent method using ethanol as solvent and CCS as a super disintegrating agent.

Table 5: The post-compression evaluation parameters of formulations

\begin{tabular}{|c|c|c|c|c|c|c|}
\hline Code & Weight variation\$ $(\mathrm{mg})$ & Hardness* $\left(\mathrm{kg} / \mathrm{cm}^{2}\right)$ & Friability" (\%) & Thickness* (mm) & Disintegration* (s) & Drug content* $(\%)$ \\
\hline F1 & $100 \pm 1.0$ & $3.50 \pm 0.47$ & $0.60 \pm 0.05$ & $3.11 \pm 0.14$ & $26.11 \pm 0.41$ & $82.31 \pm 0.77$ \\
\hline $\mathrm{F} 2$ & $100 \pm 1.12$ & $3.40 \pm 0.18$ & $0.70 \pm 0.04$ & $3.09 \pm 0.23$ & $24.45 \pm 0.52$ & $91.04 \pm 0.68$ \\
\hline F3 & $100 \pm 1.43$ & $3.30 \pm 0.43$ & $0.60 \pm 0.03$ & $3.18 \pm 0.19$ & $23.33 \pm 0.63$ & $89.39 \pm 1.75$ \\
\hline $\mathrm{F} 4$ & $100 \pm 1.44$ & $3.33 \pm 0.87$ & $0.50 \pm 0.02$ & $3.13 \pm 0.17$ & $22.59 \pm 0.55$ & $95.27 \pm 0.57$ \\
\hline F5 & $100 \pm 1.83$ & $3.29 \pm 0.50$ & $0.90 \pm 0.01$ & $3.12 \pm 0.05$ & $25.15 \pm 0.47$ & $86.02 \pm 1.95$ \\
\hline F6 & $100 \pm 1.13$ & $3.5 \pm 0.83$ & $0.80 \pm 0.03$ & $3.16 \pm 0.11$ & $27.32 \pm 0.12$ & $90.97 \pm 0.90$ \\
\hline
\end{tabular}

Results are shown as mean $\pm \operatorname{SD}(* n=3, \$ n=20$, \# $n=6)$



Fig. 7: Dissolution profile of various formulation of lamotrigine solid dispersion prepared by using ethanol

\section{CONCLUSION}

In this study, we have prepared the orally disintegrating tablet consisting of solid dispersion of lamotrigine with soya lecithin as phospholipid. Solid dispersion of soya lecithin-lamotrigine using ethanol as a solvent showed a significant increase in solubility over and above the increase in rate and extent of dissolution, which may increase the bioavailability of lamotrigine. The improved dissolution behavior of drug-phospholipid solid dispersions is expected due to the significant increase in the drug's total surface area during the dissolution process. Solid dispersion of lamotrigine with soya lecithin also showed significant antiepileptic activity as compared to pure lamotrigine. Also, optimized solid dispersion on oral administration delayed the onset of convulsions and offered complete protection against the PTZ induced seizures compared to pure lamotrigine. ODT consisting of solid dispersion exhibited fast and greater release of lamotrigine. Thus the formulation of lamotrigine solid dispersion as ODT offers promising advantages over conventional dosage with its immediate onset of action.

\section{AUTHORS CONTRIBUTIONS}

All the authors have contributed equally.

\section{CONFLICT OF INTERESTS}

The authors report no conflict of interest.

\section{REFERENCES}

1. Tan L, Yu JT, Guan HS. Intranasal anticonvulsive treatment: prospective management of intractable epilepsy? Med Hypotheses 2008;71:542-5.

2. Lamotrigine RX. LIST, drugs a-z list lamictal (lamotrigine) drug center. Lamictal (Lamotrigine drug information, drugs a-z list, lamictal (lamotrigine) drug center; 2019. 
3. Savjani KT, Gajjar AK, Savjani JK. Drug solubility: importance and enhancement techniques. ISRN Pharm; 2012. p. 1-10.

4. Vimalson DC, Parimalakrishnan S, Jeganathan NS, Anbazhagan S. Solid dispersion technique to enhance the solubility and dissolution of febuxostatan BCS class II drug. Int J Appl Pharm 2019;11:241-6.

5. Sanjaymitra PSS, Ganesh GNK. Dissolution and solubility enhancement strategies: current and novel prospectives. J Crit Rev 2018;5:1-10.

6. Goa KL, Ross SR, Chrisp P. Lamotrigine-a review of its pharmacological properties and clinical efficacy in epilepsy. Drugs 1993;46:152-76.

7. Hassib ST, Hashem HMA, Mahrouse MA, Mostafa EA. A validated reversed-phase high-performance liquid chromatography method for the simultaneous determination of five antiepileptic drugs used in the treatment of lennoxgastaut syndrome in their pharmaceutical dosage form. Asian J Pharm Clin Res 2018;11:167-73.

8. Mohan A, Rana R. Lamotrigine solid dispersions for solubility enhancement. Asian J Pharm 2016;10:172-7.

9. Kadam PA, Dherai AJ, Naik P, Lokhande R, Udani V, Gursahani $\mathrm{R}$, et al. Retrospective study on therapeutic drug monitoring of lamotrigine in Indian epileptic patients. Int J Pharm Pharm Sci 2014;6:430-3.

10. Han HK, Choi HK. Improved absorption of meloxicam via salt formation with ethanolamines. Eur J Pharm Biopharm 2007;65:99-103.

11. Taksande JB, Lade SN, Trivedi RV, Mahore JG, Umekar MJ. Effect of hydrophilic polymer on solubility and dissolution of atorvastatin inclusion complex. Int J Pharm Chem Sci 2012;1:374-85.

12. Trivedi RV, Admane PS, Taksande JB, Mahore JG, Umekar MJ. Solubility enhancement studies of hydrochlorothiazide by preparing solid dispersions using losartan potassium and urea by different methods. Der Pharm Lett 2011;3:8-17.

13. Venkat BY, Adhikrao VY. Enhancement of solubility and dissolution rate of BCS class II pharmaceuticals by non aqueous granulation technique. Int J Pharm Res Dev 2010;1:1-11.

14. Shende AJ, Patil RR, Devarajan PV. Microemulsion of lamotrigine for nasal delivery. Indian J Pharm Sci 2007;69:721-2.

15. Patil AN, Shinkar DM, Saudagar RB. Review article: solubility enhancement by solid dispersion. Int J Curr Pharm Res 2017;9:15-8.
16. Ford JL. The current status of solid dispersions. Pharm Acta Helv 1986;61:69-88.

17. Mohan A, Gundamaraju R. In vitro and in vivo evaluation of fastdissolving tablets containing solid dispersion of lamotrigine. Int J Pharm Invest 2015;5:57-64

18. Mirza S, Miroshnyk I, Habib M, Brausch J, Hussain MD. Enhanced dissolution and oral bioavailability of piroxicam formulations: modulating the effect of phospholipids. Pharmaceutics 2010;2:339-50.

19. Sosada M, Gorecki M, Pasker B. Influence of rapeseed phospholipids on ibuprofen dissolution from solid dispersions. Pharmazie 2006;61:677-80.

20. Vudathala GK, Rogers JA. Dissolution of fludrocortisone from phospholipid coprecipitates. J Pharm Sci 1992;81:282-6.

21. Serajuddin ATM. Solid dispersion of poorly water-soluble drugs, early promises, subsequent problems and recent breakthroughs. J Pharm Sci 1999;88:1058-66.

22. Li J, Wang XL, Zhang T, Wang CL, Huang ZJ, Luo X, et al. A review on phospholipids and their main applications in drug delivery systems. Asian J Pharm Sci 2015;10:81-98.

23. Van Hoogevest $P$, Wendel A. The use of natural and synthetic phospholipids as pharmaceutical excipients. Eur J Lipid Sci Technol 2014;116:1088-107.

24. Chiou WL, Riegelman S. Pharmaceutical applications of solid dispersion systems. J Pharm Sci 1971;60:1281-302.

25. Hussain D, Brausch F, Talukder M. Ibuprofen-phospholipid solid dispersions: Improved dissolution and gastric tolerance. Int J Pharm 2012;422:290-4.

26. Shinde VR, Shelake MR, Shetty SS, Chavan-Patil AB, Pore YV, Late SG. Enhanced solubility and dissolution rate of lamotrigine by inclusion complexation and solid dispersion technique. J Pharm Pharmacol 2008;60:1121-9.

27. Mali A, Nalavade S, Bathe R. Physicochemical characterization and solubility enhancement studies of felodipine solid dispersion. Int J Biomed Adv Res 2015;6:391-9.

28. Taksande JB, Wadher KJ, Trivedi RV, Umekar MJ. Development and evaluation of lamotrigine loaded N-trimethyl chitosan microspheres for intranasal administration. Int J Chem Tech Res 2017;10:1-13.

29. Taksande JB, Sonwane PP, Trivedi RV, Wadher KJ, Umekar MJ. Formulation and pharmacodynamic investigations of lamotrigine microspheres in pentylenetetrazole-induced seizures in mice. Asian J Pharm 2017;11:215-24. 